\title{
EyeArt + EyePACS: Automated Retinal Image Analysis For Diabetic Retinopathy Screening in a Telemedicine System
}

\author{
Malavika Bhaskaranand ${ }^{1 \star}$, Jorge Cuadros ${ }^{2}$, Chaithanya Ramachandra ${ }^{1}$, \\ Sandeep Bhat ${ }^{1}$, Muneeswar G. Nittala ${ }^{3}$, Srinivas R. Sadda ${ }^{3}$, and Kaushal \\ Solanki ${ }^{1}$ \\ 1 Eyenuk, Inc. Los Angeles, CA, USA, \\ 2 EyePACS LLC., San Jose, CA, USA \\ 3 Doheny Eye Institute, Los Angeles, CA, USA
}

\begin{abstract}
Telemedicine frameworks are key to screening the large, evergrowing diabetic population for preventable blindness due to diabetic retinopathy (DR). Integrating fully-automated screening systems in telemedicine frameworks will make DR screening more efficient, cost-effective, reproducible, and accessible. In this paper, we present the integration of EyeArt, an automated DR screening system, into EyePACS, a telemedicine system for DR screening used in diverse screening settings. EyeArt incorporates novel image processing and analysis algorithms for assessing image gradability; enhancing images based on median filtering; detecting interest regions and localizing lesions based on multi-scale morphological analysis; and DR screening and thus achieves robustness to the large image variability seen in a telemedicine system such as EyePACS. EyeArt is implemented as a scalable, high-throughput cloud-based system to enable large-scale DR screening. We evaluate the safety and performance of EyeArt on a dataset with 434,023 images from 54,324 patient cases obtained from EyePACS. On this dataset, EyeArt's screening sensitivity is $90 \%$ at specificity $60.8 \%$ and the area under the receiver operating characteristic curve (AUROC) is 0.883 . In a setup where trained human graders review patient cases recommended for referral by EyeArt with low confidence, a workload reduction of $62 \%$ is possible. Therefore, EyeArt can be safely integrated into large real world telemedicine DR screening programs such as EyePACS helping reduce workload and increase efficiency and thus help in reducing vision loss due to DR through early detection and treatment.
\end{abstract}

Keywords: image analysis, telemedicine, screening, diabetic retinopathy, EyeArt, EyePACS

\section{Introduction}

Diabetes mellitus is a chronic disease that impairs insulin production which in turn hampers the body's ability to metabolize glucose. The number of people with diabetes worldwide is estimated to be a staggering 387 million in 2014 [10].

\footnotetext{
* Corresponding author: malavika@eyenuk.com
}

X. Chen, M. K. Garvin, J. Liu, E. Trucco, Y. Xu (Eds.): OMIA 2015, Held in Conjunction with MICCAI 2015, Munich, Germany, Iowa Research Online, pp. 105-112, 2015. Available from: http://ir.uiowa.edu/omia/2015_Proceedings/2015/ 
With the increase in diabetes incidence and the relatively low diabetes mortality, the prevalence of diabetes is expected to increase and reach 592 million by 2035 [10].

Diabetic retinopathy (DR) is a common microvascular complication of diabetes in which blood vessels in the retina become fragile and leak. DR is the leading cause of new-onset blindness and vision loss among the working-age population in the western world [9], with approximately 24,000 people losing vision from DR each year in the US alone. Studies have demonstrated that early detection and treatment of DR helps reduce blindness and vision loss [11] and that DR screening is cost-effective [11]. Therefore, annual DR screening is recommended for diabetic patients [9].

Many diabetic patients do not get annual DR screening since DR can progress over several years without symptoms or discomfort before reaching a stage with vision loss. In addition, today eye care providers cannot meet the screening needs of the large and growing diabetic population with manual DR screening alone. In order to address the lack of adherence with yearly eye exams, DR screening programs $[6,4,1,3]$ have been developed in primary care facilities using noninvasively captured digital color fundus images that are read remotely by trained graders in a telemedicine system. EyePACS [6] is a well established telemedicine program that provides a web-based DR screening framework designed to simplify the process of image capture, transmission, and review.

A fully-automated computerized screening system used for triaging patient cases will go a long way in reducing the workload of trained graders in telemedicine programs and thus make the DR screening process more efficient, cost-effective, reproducible, and accessible. Therefore, integrating automated DR screening systems in telemedicine systems is key to meeting the fast-growing need for DR screening for preventable blindness.

Many automated programs have been developed over the years to interpret retinal images, however, they have generally been tested against standard reference retinal image databases, such as MESSIDOR [12], Diaret1db [7], or DRIVE [13], which provide ground truth in hundreds of retinal images for testing. While these image sets are generally carefully adjudicated for retinal lesions and vasculature, they may not represent the variety of retinal images that are captured in real world DR screening environments. Image interpretation algorithms that perform well on standard retinal image databases may perform inadequately when deployed in actual clinical practice where image quality, pupil size, fundus pigmentation, illumination conditions, and retinal cameras are quite variable.

Eyenuk's EyeArt program [2] is an automated high-throughput DR screening system that has performed well when tested against the EyePACS database of retinal images captured in actual clinical settings using a variety of fundus cameras. In this paper we present the integration of EyeArt, an automated DR screening system, into the EyePACS Retinal Reading Program, a telemedicine system for DR screening. We evaluate the safety and performance of EyeArt on a large color retinal fundus image dataset with 54,324 patient cases obtained from EyePACS and show that EyeArt achieves a high sensitivity of $90 \%$ at speci- 
ficity of $60.8 \%$ for identifying patients requiring referral with AUROC of 0.883 . EyeArt's excellent performance on the EyePACS dataset with large variability across images can be primarily attributed to the novel image processing and analysis algorithms used for assessing gradability of images; enhancing images; segmenting and localizing lesions; and DR screening based on all images in a patient visit.

\section{System Design and Approach}

\subsection{EyePACS telemedicine system}

Primary care clinic personnel (nursing, technical, or administrative staff) are trained and certified by the EyePACS program coordinators to acquire retinal images from standard digital retinal cameras. Relevant clinical data and eight high-resolution images per patient (two external and 6 retinal images) are encrypted and transmitted to a secure server using a standard computer and web browser. Images are then interpreted by certified EyePACS reviewers or local eye care providers who are certified through the EyePACS Retinopathy Grading System (ERGS). Reports indicating retinopathy level and referral recommendations are transmitted back to primary care providers through the EyePACS website or through interfaces between EyePACS and Health Level 7-compliant electronic medical records or chronic disease registries.

EyePACS uses an image evaluation protocol, the EyePACS Retinopathy Grading System (ERGS), developed by Bresnick [6] and based on the Early Treatment Diabetic Retinopathy Study (ETDRS) [8]. The protocol evaluates the presence and severity of the discrete retinal lesions associated with diabetic retinopathy. The lesions graded are shown in Figure 1. In addition, the presence or absence of laser scars (focal and panretinal) is graded. Readers grade each lesion type separately in each eye using an online grading template which records a choice for each lesion type among: absent ["no"], present ["yes"] or ["cannot grade"].

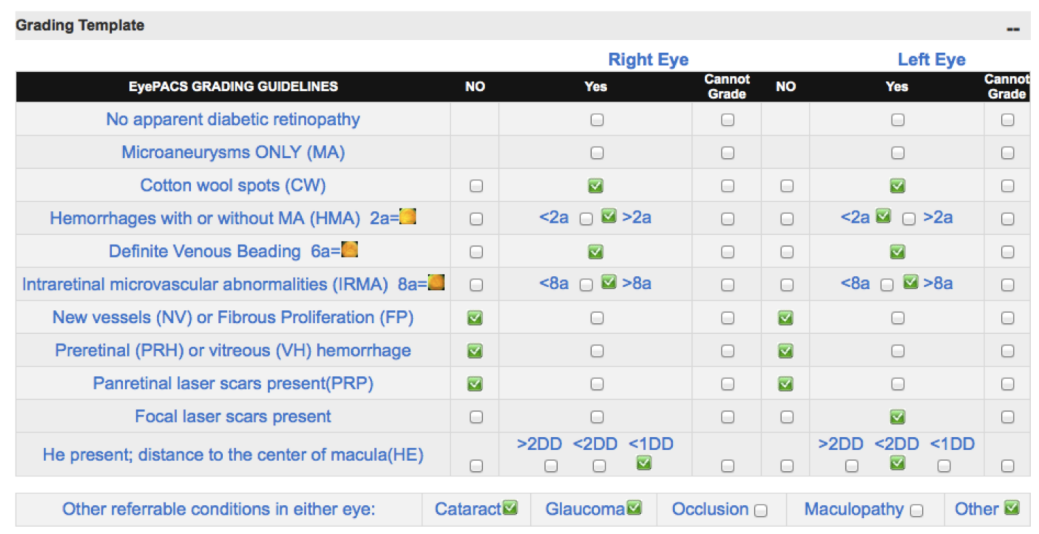

Fig. 1. EyePACS grading template 
Based on the severity of discrete retinal lesions, each patient visit is assigned a DR severity level as per the International Clinical Diabetic Retinopathy (ICDR) scale [5] with 0 for no apparent DR; 1 for mild non-proliferative DR (NPDR); 2 for moderate NPDR; 3 for severe NPDR; and 4 for proliferative DR (PDR), and a Macular Edema (ME) level with 0 for no ME and 1 for ME. A patient was deemed to have non-referable DR if there was no NPDR or mild NPDR and no $\mathrm{ME}$ in both eyes.

\subsection{EyeArt - Automated DR screening}

EyeArt is a computerized screening tool developed by Eyenuk, Inc. that assesses the severity of DR based on multiple color fundus images captured during a patient visit or encounter, and provides a recommendation for referral to an eye-care specialist if the patient is deemed to have ME or moderate NPDR or higher on the ICDR scale. The primary output of EyeArt is a "refer" or "no refer" screening recommendation. EyeArt is designed to provide a "refer" recommendation when it detects signs of moderate NPDR or higher on the ICDR scale; detects signs of ME; or deems the images as ungradable. EyeArt outputs a decision statistic that can be used to assess the confidence in the recommendation. In addition, EyeArt provides a "confident refer" recommendation using thresholds at $98 \%$ specificity.

The overall block diagram of the EyeArt system is shown in Figure 2. The patient encounter comprising multiple color fundus images is first analyzed for gradability. Non-retinal images such as those of the external image of the eye, retinal images with poor quality due to various factors such as poor illumination, over-exposure and poor focus are excluded from further analysis. If the patient encounter has at least one retinal image with acceptable quality per eye, it is deemed as gradable. Non-gradable patient encounters are not analyzed and flagged as having insufficient data.

Image enhancement: Retinal fundus images have different colors levels, different dynamic range, and different sensor noise. Image enhancement is necessary to normalize the appearance and enhance the appearance of lesions (See figure 3). For enhancement, the gradable images are first subjected to an edgepreserving bilateral filter [14] in order to remove noise without affecting the important landmarks such as lesions and vessels.

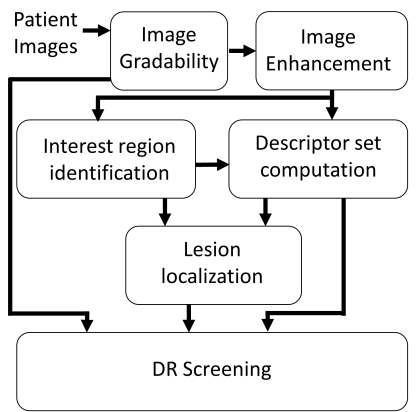

Fig. 2. Overall block diagram of EyeArt DR screening system 
The median normalized image intensity $I_{\mathrm{Norm}, \mathcal{S}}$ at pixel location $(x, y)$ is computed as,

$I_{\mathrm{Norm}, \mathcal{S}}(x, y)= \begin{cases}C_{\mathrm{mid}}+\left(C_{\mathrm{mid}}-1\right) \cdot \frac{I(x, y)-I_{\mathrm{Back}, \mathcal{S}}(x, y)}{C_{\mathrm{max}}-I_{\mathrm{Back}, \mathcal{S}}(x, y)} & \text { if } I(x, y) \geq I_{\mathrm{Back}, \mathcal{S}}(x, y), \\ C_{\mathrm{mid}} \cdot \frac{I(x, y)}{I_{\mathrm{Back}, \mathcal{S}}(x, y)} & \text { otherwise }\end{cases}$

where $I$ is the input image with pixel intensities in the range $\left[C_{\min }, C_{\max }\right]=$ $\left[0,2^{B}-1\right], B$ is the image bit-depth, $I_{\text {Back } \mathcal{S}}$ is background image obtained using a median filter over the area $\mathcal{S}$, and $C_{\text {mid }}=2^{B-1}$ is the "middle" gray pixel intensity value in image $I$. For an 8-bit image, $\left[C_{\min }, C_{\max }\right]=[0,255]$, and $C_{\text {mid }}=128$. Empirically, $\mathcal{S}$ is chosen to be a circle of radius $\mathrm{r}=100$.

Interest region identification: The enhanced images are then analyzed to identify regions of the image potentially containing interesting anatomical or pathological structures. The interest region detector based on multi-scale morphological filterbank analysis that identifies putative lesion-specific interest regions (See figure 4).

Descriptor computation: The pixels identified by the interest region detector are described using a set of feature descriptors (multi-scale median filterbank descriptors, oriented median filterbank descriptors, and other established descriptors) that allow local description at multiple scales. The interesting pixels are classified using supervised learning classifiers into lesion types (See figure 5).

DR screening classification: The pixel-level classifier decision statistics are averaged within each lesion to obtain the lesion-level decision statistics. The histograms of lesion-level decision statistics for different lesion types are collated to generate the image-level descriptor. The image-level descriptors for all the gradable images in a patient encounter/visit are combined to generate an encounter-level descriptor that is classified using a supervised learning ensemble classifier to detect the presence of moderate NPDR or higher on the ICDR scale.

\subsection{Integration of EyeArt into EyePACS}

The EyeArt interface has been designed to be REST-ful and hence can be accessed via standard HTTPS requests. The design for the integration of EyeArt automated screening system into the EyePACS telemedicine screening framework is shown in Figure 6. The patient images are passed on to the EyeArt

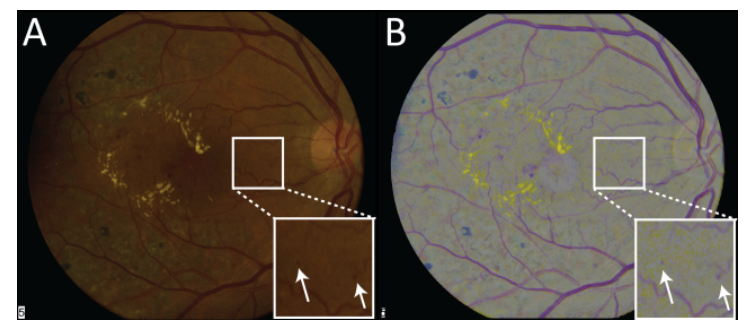

Fig. 3. Improved lesion and vessel visibility after enhancement. The inset shows enhanced microaneurysms. 

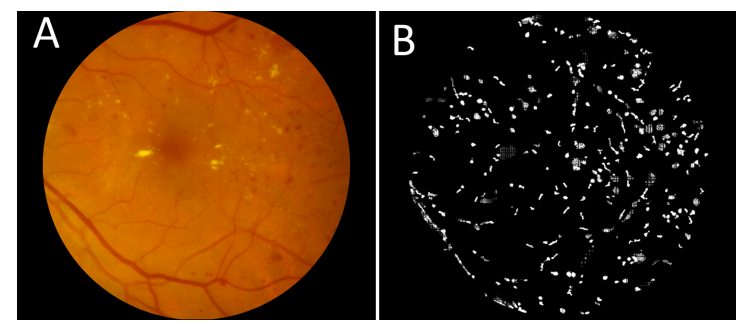

Fig. 4. Examples of interest regions identification. A: Original image showing lesions (bright yellow and dark red spots). B: Binary map showing interesting pixels forming less than $1 \%$ of the total pixels in the image.

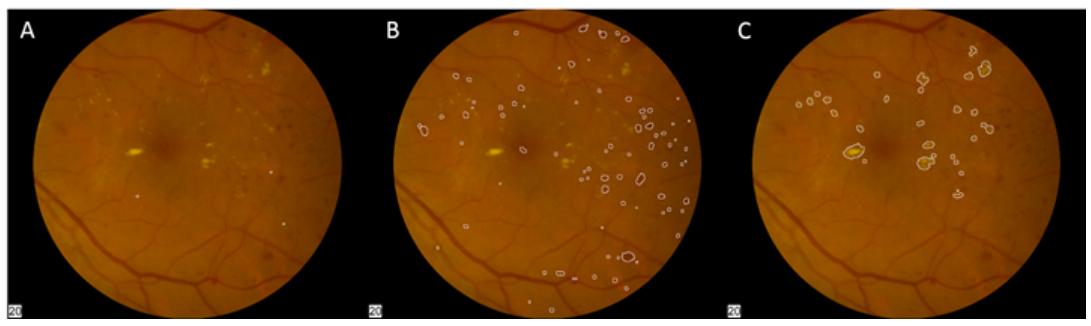

Fig. 5. Example of fully-automated lesion localization and segmentation by EyeArt. A: Microaneurysms B: Hemorrhages. C: Exudates. Most of these lesions in this example retinal image are correctly detected and localized.

system from EyePACS via a HTTPS POST request after removal of patient identifying data and assigning a unique patient ID. The EyePACS system pings the EyeArt system via HTTPS GET requests for results of the patient encounter which are provided when available.

\section{Dataset and results}

The EyePACS dataset included a total of 434,023 color retinal fundus images of 54,324 diabetic patient visits or encounters without any patient identification data. Each of these encounters had 2-24 images including external lens-shots. These deidentified images were captured between April 2006 and December 2013 at various DR screening centers that use the EyePACS Retinal Reading Program.

The prevalence of the various DR levels on the ICDR scale and ME as per the gold standard grading provided by EyePACS graders is tabulated in Table 1. A total of 8,189 cases (15.1\% of the cases) were deemed to have referable DR (moderate NPDR or higher); 1,965 cases (3.6\% of the cases) were deemed to have treatable DR (severe NPDR or higher) and 2,339 cases (4.3\% of the cases) were deemed to have ME. No case was deemed by graders to be ungradable due to poor quality.

All the 54,324 encounters from the EyePACS dataset were processed by EyeArt in about 24 hours. EyeArt marks 500 encounters (0.9\%) as non-gradable and these encounters are considered to have a "refer" recommendation for the performance analysis. EyeArt screening sensitivity is 90\% (95\% CI: 89.4\% - 90.6\%) at specificity of $60.8 \%$ (95\% CI: $60.4 \%-61.3 \%$ ) at an operating point of -0.875 


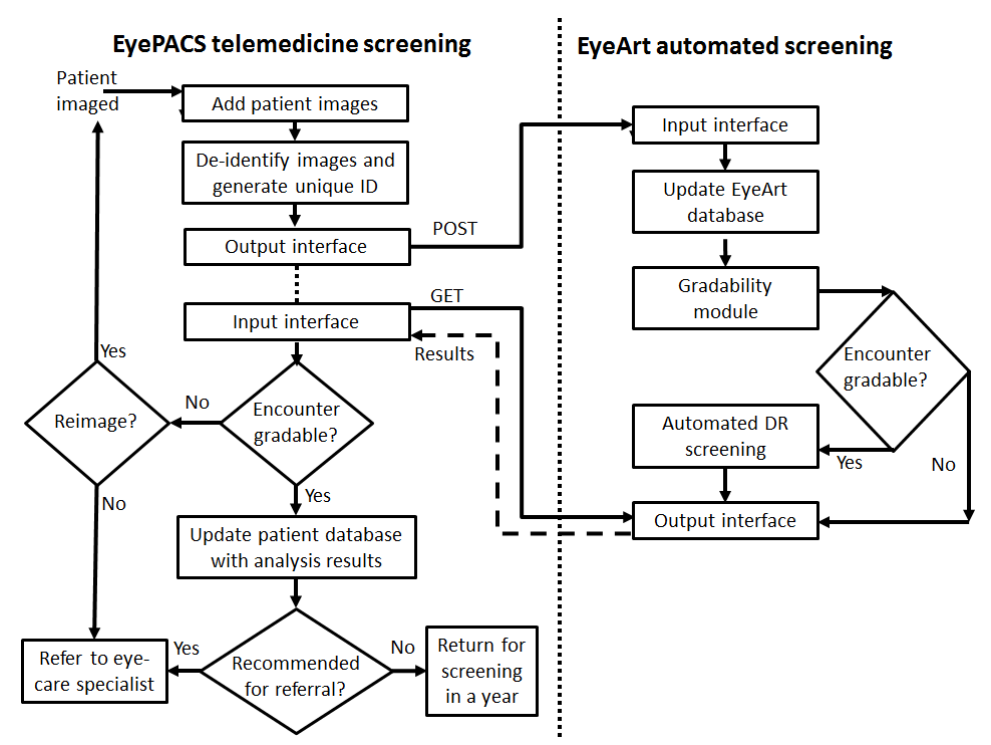

Fig. 6. Interface between the EyePACS telemedicine screening system and the EyeArt automated screening tool.

on the decision statistic. This corresponds to 25,491 "refer" recommendations, 4,830 of which are "confident refer" recommendations with high confidence i.e. at $98 \%$ specificity thresholds. The AUROC is 0.883 (95\% CI: $0.878-0.887)$. As shown in Table 2, there are 828 false negatives (only $1.5 \%$ of the cases) out of which 724 do not meet the treatment criteria [5] i.e. have moderate NPDR and no macular edema. A careful analysis of the false negative cases reveals that most of the misses are either in cases of questionable image quality or where the missed lesions are very faint.

The screening sensitivity of EyeArt at reasonable specificity values is better than the sensitivities achieved by human graders. If all the 25,491 cases in the EyePACS dataset for which EyeArt provides a "refer" recommendation are reviewed by human graders, the graders' workload is reduced by $53 \%$ compared to the system without EyeArt where they see all patient images. Of the 25,491

Table 1. Distribution of DR levels and ME levels in the EyePACS dataset as per gold standard grading

\begin{tabular}{|l|r|r|r|}
\hline \multirow{2}{*}{ ICDR scale } & \multicolumn{2}{|c|}{ Number of cases (Percentage of cases) } \\
\cline { 2 - 4 } & Without ME & With ME & Total \\
\hline No apparent DR (0) & $40683(74.9)$ & $0(0.0)$ & $40683(74.9)$ \\
Mild NPDR (1) & $5359(9.9)$ & $14(0.0)$ & $5373(9.9)$ \\
Moderate NPDR (2) & $4922(9.1)$ & $1302(2.4)$ & $6224(11.5)$ \\
Severe NPDR (3) & $560(1.0)$ & $576(1.1)$ & $1136(2.1)$ \\
Proliferative DR (4) & $461(0.8)$ & $368(0.7)$ & $829(1.5)$ \\
DR level unknown (-1) & $0(0.0)$ & $79(1.4)$ & $79(1.4)$ \\
Ungradable due to poor quality & $0(0.0)$ & $0(0.0)$ & $0(0.0)$ \\
\hline
\end{tabular}


112 M. Bhaskaranand et al.

Table 2. Analysis of EyeArt false negatives in the EyePACS dataset with 54,324 cases. The 724 missed cases with moderate NPDR and no ME do not meet treatment criteria.

\begin{tabular}{|l|l|l|l|}
\hline \multirow{2}{*}{ ICDR scale } & \multicolumn{3}{|c|}{ Number of false negatives } \\
\cline { 2 - 4 } & Without ME & With ME & Total \\
\hline Moderate NPDR (2) & 724 & 72 & 796 \\
Severe NPDR (3) & 10 & 4 & 14 \\
PDR (4) & 17 & 1 & 18 \\
\hline
\end{tabular}

"refer" recommendations, if the 4,830 "confident refer" recommendations are not seen by graders, their workload is reduced by $62 \%$ (or by factor of 2.6 ).

\section{Conclusion}

EyeArt achieves high sensitivity (90\% at $60.8 \%$ specificity) for identifying patients with referable DR on 434,023 retinal images of 54,324 patients from the EyePACS telescreening system. Our analysis also shows that EyeArt can provide a workload reduction of $62 \%$. To the best of our knowledge, this is the first time an automated DR screening system has been validated on a large, realworld dataset with more than 50,000 patient visits. The results demonstrate that EyeArt can be safely integrated into image reading centers within telemedicine frameworks and can help increase efficiency and cost-savings by reducing the workload of trained graders. Automated retinal image analysis for DR screening integrated into telemedicine systems has the potential to greatly reduce the burden on DR screening systems by improving health care productivity and thus help in reducing vision loss due to DR through early detection and treatment.

\section{References}

1. CARA, www.diagnos.ca/cara/CARA_in_6_steps-en-39, Accessed: July 312015

2. EyeArt, http://www.eyenuk. com/eyeart.html, Accessed: July 312015

3. IRIS, www.retinalscreenings.com, Accessed: July 312015

4. Agurto, C., Murray, V., Barriga, E., Murillo, S., Pattichis, M., Davis, H., Russell, S., Abrmoff, M., Soliz, P.: Multiscale AM-FM methods for diabetic retinopathy lesion detection. IEEE Trans. Medical Imaging 29(2), 502-512 (2010)

5. American Academy of Ophthalmology: International Clinical DR disease severity scale (May 2010)

6. Cuadros, J., Bresnick, G.: EyePACS: an adaptable telemedicine system for diabetic retinopathy screening. J. of Diab. Sci. and Tech. 3(3), 509-516 (May 2009)

7. Diaret1db: DR1 dataset (Mar 2014), dx.doi.org/10.6084/m9.figshare. 953671

8. ETDRS research group: Fundus Photographic Risk Factors for Progression of Diabetic Retinopathy: ETDRS Rep.\#12. Ophth. 98(5, Supp.), 823-833 (May 1991)

9. Fong, D.S., Aiello, L.P., Ferris, F.L., Klein, R.: Diabetic Retinopathy. Diabetes Care 27(10), 2540-2553 (Oct 2004)

10. International Diabetes Federation: Diabetes Atlas, www.idf.org/diabetesatlas

11. Klonoff, D.C., Schwartz, D.M.: An economic analysis of interventions for diabetes. Diabetes Care 23(3), 390-404 (Mar 2000)

12. Messidor: Retinal Image Databases http://messidor.crihan.fr/index-en.php

13. Staal, J., Abramoff, M., Niemeijer et al., M.: Ridge based vessel segmentation in color images of the retina. IEEE Trans. Medical Imaging 23(4), 501-509 (2004)

14. Tomasi, C., Manduchi, R.: Bilateral filtering for gray and color images. In: Comp. Vis., 1998. Sixth Int. Conf. on. pp. 839-846 (1998) 